\title{
Phase-contrast helium-3 MRI of aerosol deposition in human airways
}

\author{
Mathieu Sarracanie ${ }^{a, b, c *}$, Denis Grebenkov ${ }^{d}$, Julien Sandeau e,f \\ Soulé Coulibaly ${ }^{a}$, Andrew R. Martine, Kyle Hill ${ }^{a, g}$, \\ José Manuel Pérez Sánchez ${ }^{\mathrm{a}}$, Redouane Fodil ${ }^{\mathrm{f}}$, Lionel Martin ${ }^{\mathrm{a}}$, \\ Emmanuel Durand ${ }^{a}$, Georges Caillibotte ${ }^{e}$, Daniel Isabey ${ }^{f}$, Luc Darrasse ${ }^{a}$, \\ Jacques Bittoun ${ }^{a}$ and Xavier Maître ${ }^{a}$
}

\begin{abstract}
One of the key challenges in the study of health-related aerosols is predicting and monitoring sites of particle deposition in the respiratory tract. The potential health risks of ambient exposure to environmental or workplace aerosols and the beneficial effects of medical aerosols are strongly influenced by the site of aerosol deposition along the respiratory tract. Nuclear medicine is the only current modality that combines quantification and regional localization of aerosol deposition, and this technique remains limited by its spatial and temporal resolutions and by patient exposure to radiation. Recent work in MRI has shed light on techniques to quantify micro-sized magnetic particles in living bodies by the measurement of associated static magnetic field variations. With regard to lung MRI, hyperpolarized helium-3 may be used as a tracer gas to compensate for the lack of MR signal in the airways, so as to allow assessment of pulmonary function and morphology. The extrathoracic region of the human respiratory system plays a critical role in determining aerosol deposition patterns, as it acts as a filter upstream from the lungs. In the present work, aerosol deposition in a mouth-throat phantom was measured using helium-3 MRI and compared with single-photon emission computed tomography. By providing high sensitivity with high spatial and temporal resolutions, phase-contrast helium-3 MRI offers new insights for the study of particle transport and deposition. Copyright @ 2014 John Wiley \& Sons, Ltd.
\end{abstract}

Keywords: aerosol deposition; drug administration; human airways; helium-3 MRI; SPECT

\section{INTRODUCTION}

The impact of inhaled aerosols on human health is widespread, ranging from the risks associated with environmental or workplace aerosol exposure to the beneficial effects of medical aerosols. Aerosol drug delivery targets a wide range of applications from local airway treatment of pulmonary diseases to wholebody drug administration following systemic circulation. In the latter case, the very large exchange surface area of the lungs

* Correspondence to: M. Sarracanie, Imagerie par Résonance Magnétique Médicale et Multi-Modalités (UMR8081), IR4M, Université Paris-Sud, CNRS, Orsay, France.

E-mail: msarraca@physics.harvard.edu

a M. Sarracanie, S. Coulibaly, K. Hill, J. M. Pérez Sánchez, L. Martin, E. Durand, L., Darrasse, J. Bittoun, X. Maître

Imagerie par Résonance Magnétique Médicale et Multi-Modalités (UMR8081), IR4M, Université Paris-Sud, CNRS, Orsay, France

b M. Sarracanie

Department of Physics, Harvard University, Cambridge, MA, USA

c M. Sarracanie

MGH/A. A. Martinos Center for Biomedical Imaging, Boston, MA, USA

d D. Grebenkov

Laboratoire de Physique de la Matière Condensée, Ecole Polytechnique, CNRS,

Palaiseau, France combined with a reduced metabolic activity with respect to that of the gastrointestinal tract, a thin alveolar epithelium in the deeper airways, and a dense blood supply ensure adequate pulmonary bioavailability and rapid drug absorption $(1,2)$. Additionally, drug administration through the natural oronasal route is minimally invasive and painless. This approach could well replace or complement many existing and widespread drug administration methods. However, drug distribution in a complex geometry such as the human respiratory tract highly depends on aerosol properties and administration parameters $(3,4)$. The tangled mechanisms of particle transport and deposition in human airways feature a series of basic phenomena. They involve

\footnotetext{
e J. Sandeau, A. R. Martin, G. Caillibotte

Centre de Recherche Claude Delorme (CRCD), Air Liquide, Les Loges-en-Josas, France

f J. Sandeau, R. Fodil, D. Isabey

Biomécanique Cellulaire et Respiratoire, IMRB (UMR955), INSERM, UPEC, CNRS, Créteil, France

g K. Hill

Radiology Research Group, Oxford MRI Centre, University of Oxford, Oxford, UK
}

Abbreviations used: $C T$, computed tomography; FWHM, full width at half maximum; HRCT, high-resolution computed tomography; MMAD, mass median aerodynamic diameter; ppm, parts per million; SPECT, single-photon emission computed tomography; SNR, signal to noise ratio. 
three-phase flows, fluid-structure coupling, and heat and mass transfers through constantly changing irregular geometries. They may be partially modeled by computational fluid dynamics, but this requires experimental guidance and validation. Thus, physical methods for quantifying and locating aerosol deposition at targeted sites are needed to control and optimize the delivery of inhaled drugs. Most published studies have quantified total aerosol deposition in vitro and in vivo using biochemical assay (5), photometry (6) in the case of bolus dispersion, as well as spectrophotometric (7) and pharmacokinetic measurements (8). Nuclear medicine techniques are currently the only in vivo modalities that provide both aerosol quantification and regional localization $(5,9,10)$ but they remain limited in terms of spatial and temporal resolutions as well as by patient exposure to radiation.

Recent work in the field of MRI aims to achieve absolute particle quantification either by measuring the relaxation times of the total magnetic moment or by mapping the static magnetic field perturbations in the presence of superparamagnetic iron oxide markers $(11,12)$. Iron-based markers placed in the magnetic field of an MRI unit generate dipolar fields that locally modify the magnetic field, which typically leads to regions of low MR signal amplitude (13-15). The magnetic field perturbations induced by the presence of iron are embedded in the phase of the MR signal and can be processed to obtain absolute quantification of the iron mass distribution (12,16-18). For lung imaging though, standard MRI is impaired by the low tissue density of the organ and the susceptibility differences at the air-tissue interface. Experiments by Martin et al. in mice were performed postmortem, requiring a $30 \mathrm{~min}$ acquisition time (11). For in vivo work, provided the particle distribution is not affected over several respiratory cycles, control over the subject's ventilation and/or respiratory gating would be required to permit such a long acquisition time. To circumvent the limitations of standard MRI, hyperpolarized helium-3 MRI has been developed for probing the organ morphology and function (19-30). Hyperpolarized helium-3 MRI is free from ionizing radiation and provides millimeter and sub-second resolutions with high signal to noise ratios (SNRs) such that static and dynamic ventilation acquisitions can be performed during a single breath hold or a respiratory cycle (31).

Before reaching the lung, inhaled aerosol is first filtered by the extrathoracic region. Deposition in this region has been extensively studied in vitro by radionuclide scanning techniques $(32,33)$. The present work demonstrates a new method to quantify particle deposition using phase-contrast hyperpolarized helium-3 MRI in a realistic, rigid human mouth-throat phantom. The aerosol used was doubly labeled with superparamagnetic iron oxide and radioisotope technetium-99 $\mathrm{m}$ to permit detection by both helium-3 MRI and single-photon emission computed tomography (SPECT).

\section{MATERIALS AND METHODS}

\section{Aerosol administration in a mouth-trachea phantom}

\section{Design of the mouth-trachea phantom}

The main human anatomical regions between the mouth and trachea are featured on the central slice of a high-resolution computed tomography (HRCT) scan in Fig. 1(a). A 3D triangular mesh of the phantom geometry was generated from the assembly of two elements (34): the human dental print of a $50 \%$ open mouth was used for the oral cavity (Fig. 1(b), (1)) and the HRCT scan for the oropharynx, larynx, and trachea (Fig. 1(b), (2)). The model was segmented and 22 sections were defined from the inlet to the outlet, so that connecting each geometric center gave an estimated mean pathway of $220 \mathrm{~mm}$ inside the cast. After segmentation, the model was processed to have thinner mesh cells and then was built from epoxy material using stereolithography (Fig. 1(c)). All experiments in this work used this single model geometry.

\section{Preparation of the aerosol solution}

A double-labeled solution was prepared to ensure the same aerosol deposition pattern for measurement by both SPECT and MRI. Radiolabeling was done by diluting $1 \mathrm{~mL}$ technetium$99 \mathrm{~m}$ of activity $2.5 \mathrm{GBq}$ into $200 \mathrm{~mL}$ saline $(0.9 \% \mathrm{NaCl})$. MR labeling was obtained by adding $5.6 \mathrm{~mL}$ ferucarbotran $\left(\right.$ Cliavist $^{\circledR}$, Schering, Berlin, Germany). The MR contrast agent ferucarbotran is a superparamagnetic iron oxide composed of $4.2 \mathrm{~nm}$ crystalline non-stoichiometric $\mathrm{Fe}^{2+}$ and $\mathrm{Fe}^{3+}$ iron oxide cores with stabilizing carboxydextran organic polymer coating. This coating ensures aqueous solubility of the contrast agent. The resulting iron concentration in the solution was $[\mathrm{Fe}]=$ $(13.6 \pm 0.1) \mathrm{mmol} \cdot \mathrm{L}^{-1}$ and thus the iron mass concentration was $(0.784 \pm 0.004) \mathrm{g} \cdot \mathrm{L}^{-1}$.

\section{Characterization of the nebulized aerosol}

A major factor affecting deposition patterns within a particular geometry is the size distribution of the inhaled aerosol. Particle size was determined for the specific experimental setup, namely size measurements were made at the outlet of piping that was positioned between the nebulizer and phantom during the deposition experiments. Characterization of the nebulized aerosol was achieved by laser diffraction (Helos/BF with Inhaler dispersion module, Sympatec, Clausthal, Germany). As the small concentrations of radiolabel and contrast agent were not expected to influence particle sizes, these were not included during the laser diffraction measurements. Three series of 10 measurements
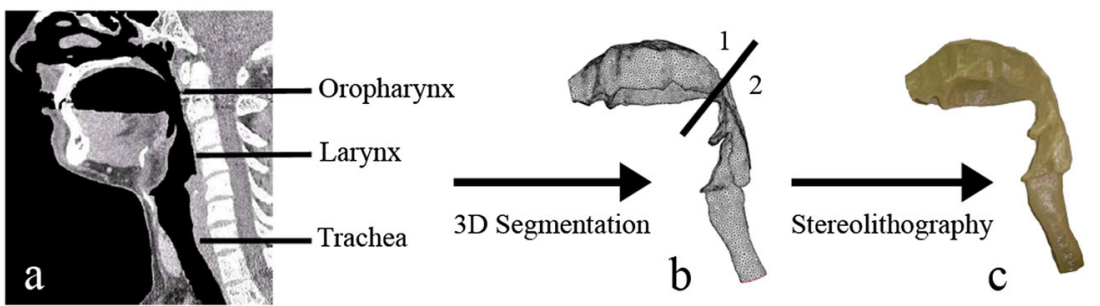

Figure 1. (a) Central slice of human HRCT head scan showing main human anatomical regions between the mouth and the trachea; (b) 3D mouththroat meshed model resulting from the assembly of a segmented human dental print (1) and HRCT scan (2); (c) mouth-throat cast built from epoxy material using stereolithography. 
(60 s each) were made. Assuming a mass density of the nebulized solution near unity $\left(1 \mathrm{~g} \cdot \mathrm{cm}^{-3}\right)$, the measured volume median diameter was estimated to be equal to the mass median aerodynamic diameter (MMAD).

\section{Administration of the nebulized aerosol}

Only $21 \%$ of the homogenous solution was nebulized over 10 min using an ultrasonic device $\left(35-\mathrm{B}^{\circledR}\right.$, DeVilbiss, Washington, DC, USA) such that the administered dose of radiolabel remained compatible with approved in vivo protocols for human ventilation imaging. The aerosol stream from the nebulizer was mixed with ambient air to achieve a total flow rate of $Q=(30 \pm 0.15)$ $\mathrm{L} \cdot \mathrm{min}^{-1}$, which is in the normal range of human breathing and similar to the experimental conditions of the work of Grgic et al. (32). The flow rate was calibrated with a mini-Buck Calibrator M-5 (Buck, Orlando, FL, USA) and regulated with a constant flow pump Bravo H Plus (TCR Tecora, Corsico, Milan, Italy). The outlet of the mouth-trachea cast was attached to four in-line breathing filters (Clear-Guard II, Intersurgical, Wokingham, UK) before being connected to a vacuum pump. A schematic diagram of the setup is shown in Fig. 2.

\section{Single-photon emission computed tomography}

Three-dimensional maps throughout the phantom were acquired immediately after aerosol administration by SPECT-CT (Symbia $^{\circledR}$, Siemens Medical, Germany). Total acquisition time was $32 \mathrm{~min}$. Counting time per view was set to $30 \mathrm{~s}$ with a total view number of 64 and isotropic voxel size of $(2.4 \times 2.4 \times 2.4)$ $\mathrm{mm}^{3}$. The actual spatial resolution of the SPECT images is however given by the point-spread function of the measuring system, here $11 \mathrm{~mm}$ full width at half maximum (FWHM). Radioactive decay corrections were applied to the raw data. The scanner was calibrated beforehand with a gamma counter by correlating the activity measured in a solution at different dilution factors with the recorded counts. Iron deposition (in $\mu \mathrm{g}$ of iron) was calculated by assuming that technetium- $99 \mathrm{~m}$ activity at a given location was proportional to the deposited aerosol. Thus, the amount of nebulized solution deposited along the phantom walls was inferred from the number of counts recorded by the gamma camera. The concentration of iron oxide in the nebulized solution being known, the absolute amount of deposited aerosol was quantified in $\mu \mathrm{g}$ of iron from the SPECT dataset. Hyperpolarized helium-3 MRI was performed in the mouththroat phantom two days after aerosol administration, allowing activity decay of the radiolabel.

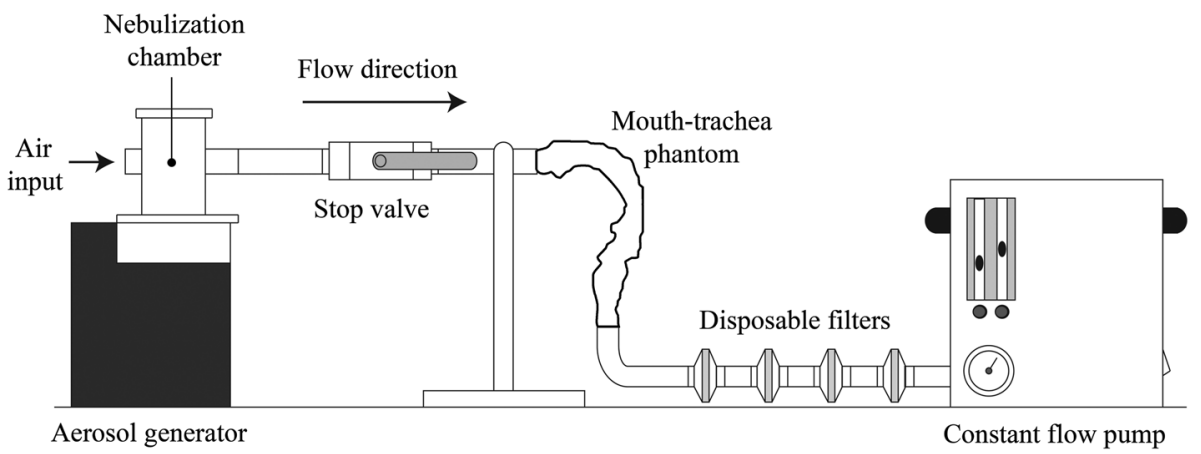

Figure 2. Schematic diagram of the experimental deposition setup.

\section{Magnetic resonance imaging}

\section{Preparation of the polarized gas mixture}

Helium-3 nuclei were dynamically hyperpolarized by metastability-exchange optical pumping with a home built polarizer (35). While flowing at $2 \mathrm{~mL} \cdot \mathrm{bar} \cdot \mathrm{min}^{-1}$ in a $1.5 \mathrm{mT}$ directing magnetic field through a $2 \mathrm{mbar} 50 \mathrm{~cm}$ long optical cell, helium-3 atoms were prepared in the metastable state $2^{3} \mathrm{~S}_{1}$ by a $2 \mathrm{MHz}$ radiofrequency discharge and electronically polarized by optical pumping to the $2^{3} \mathrm{P}_{0}$ state with a circularly polarized $2 \mathrm{~W}$ laser at $1.083 \mu \mathrm{m}$ (Keopsys, Lannion, France). Thanks to hyperfine coupling, the nuclear spin of the electronically polarized metastable helium-3 atoms was polarized accordingly. Collisions with ground state atoms where spin and metastability were conserved resulted in nuclearly polarized helium-3 atoms, which were extracted by a custom made peristaltic compressor and accumulated in a storage cell. Helium-3 doses of $15 \mathrm{~mL} \cdot$ bar, polarized at $10 \%$, were completed with $110 \mathrm{~mL} \cdot$ bar of nitrogen for each MRI acquisition.

\section{Data acquisition}

All MRI acquisitions were performed at $1.5 \mathrm{~T}$ (Achieva ${ }^{\circledR}$, Philips Healthcare, The Netherlands). Phase maps acquired in the presence of iron labeled aerosol were obtained using a 3D gradientecho sequence with four interleaved echoes of increasing echo time $\mathrm{TE}_{\{1,2,3,4\}}=\{2.6,12.6,22.6,32.6\} \mathrm{ms}$, and $\mathrm{TR}=36.5 \mathrm{~ms}$. The four echo times were chosen to explore different phase dynamic ranges in order to eventually optimize the measurement sensitivity. The full four-echo sequence was subsequently repeated three additional times. The field of view was $(288 \times 52 \times 48) \mathrm{mm}^{3}$ and the matrix size was $(72 \times 13 \times 12)$, resulting in an isotropic spatial resolution of $4 \mathrm{~mm}$. The flip angle was $\alpha=2^{\circ}$, and the bandwidth $499 \mathrm{~Hz} /$ pixel. The overall total acquisition time was $68 \mathrm{~s}$. Thereafter the deposited aerosol was fully washed from the cast under running water before being dried with compressed air and repositioned in the MRI scanner to acquire a set of reference images with the same sequence parameters. Reference phase maps were extracted. A mask was set by thresholding the 3D amplitude images at three times the standard deviation, $\sigma$, over real and imaginary noise data. Maximal SNRs were calculated for both iron weighted and reference data from maximal signal amplitudes within the masked images and the standard deviations, $\sigma$. The corresponding phase errors were computed.

\section{Magnetic field perturbation mapping}

$\mathrm{TE}_{1}$ phase maps were subtracted from $\mathrm{TE}_{\{2,3,4\}}$ for both ironweighted and reference images to produce phase shift maps 
for $\Delta \mathrm{TE}_{\{1,2,3\}}=\{10,20,30\} \mathrm{ms}$. Only the phase shift maps from the longest echo time dataset $\left(\Delta \mathrm{TE}_{3}=30 \mathrm{~ms}\right)$ were processed, as they provided the highest sensitivity without phase aliasing. Reference phase shift maps were then subtracted from ironweighted phase shift maps to account for the influence of spin dephasing processes resulting from sources other than deposited iron. The resulting phase shifts, $\Delta \Phi_{3}$, are directly proportional to the first-order static magnetic field perturbations, which are along the magnet bore $z$ axis, $\delta B_{z}(\mathbf{r})$, and induced by iron deposition integrated over the time interval $\Delta \mathrm{TE}_{3}$ :

$$
\Delta \Phi_{3} \cong \int_{\Delta \mathrm{TE}_{3}} \gamma \delta B_{z}(\mathbf{r}) \cdot \mathrm{d} t
$$

where $\gamma$ is the gyromagnetic ratio for helium-3. Deposited iron mass was quantified from the MR static magnetic field perturbation maps, $\delta B_{z}(\mathbf{r})$, extracted from Equation [1].

\section{Relaxation times and gas diffusion}

The longitudinal relaxation times, $T_{1}$, of the total helium-3 magnetic moment in the phantom in the presence and in the absence of iron oxide were estimated over the three successive scans at $10 \mathrm{~s}, 31.5 \mathrm{~s}$, and $53 \mathrm{~s}$ by fitting the echo integrals corrected for the RF depolarization with an exponential decay. The effective transverse relaxation time, $T_{2}{ }^{*}$, was mapped throughout the phantom by fitting the signal decay with an exponential over the first four interleaved echoes in the presence and in the absence of iron oxide labeled aerosol. $T_{2}{ }^{*}$ mean values and standard deviations over the phantom were calculated to determine the signal lifetimes for the two sets of acquisitions, in the presence and in the absence of iron oxide.

The fast free diffusion of helium-3 atoms in nitrogen buffer gas at $293 \mathrm{~K}$, characterized by $D \cong 7.8 \times 10^{-5} \mathrm{~m}^{2} \cdot \mathrm{s}^{-1}$ (36), led to a diffusion length over $\Delta \mathrm{TE}_{3}$ of $3.7 \mathrm{~mm}$, which remained smaller than the image spatial resolution. The dispersion effect of the gas diffusion in the phase encoding gradients may also be neglected, as the duration of these gradients was smaller than $0.6 \mathrm{~ms}$ and the related diffusion length was close to $0.3 \mathrm{~mm}$. It may also be neglected in the magnetic field perturbations resulting from the iron labeled aerosol deposition, as the latter leads to a maximal local gradient of $0.185 \mathrm{mT} \cdot \mathrm{m}^{-1}$ for the nearest neighboring voxels, which remains much smaller than the imaging magnetic field gradients.

Following P. T. Callaghan and C. D. Eccles (37), it is during the application of the reading gradient, $G_{x}$ over $T_{M}$ that the spatial resolution of the acquired images may be impaired by diffusion smearing. Hence, the evolution of the signal is governed not only by $T_{2}^{*}$ relaxation, $\exp \left(-\Delta T_{\mathrm{E} 3} / T_{2}^{*}\right)$, but also by the gas diffusion, $\exp \left[-\left(\frac{\gamma^{2} G_{x}^{2} D T_{M}^{3}}{3}\right)\right]$, such that the point-spread function of the MRI acquisition is degraded. Yet, here, its effective FWHM was not really affected by this diffusion and $T_{2}^{*}$ filtering. For the worst case, in the presence of iron oxide, $\left\langle T_{2}^{*}\right\rangle=52 \mathrm{~ms}, T_{M}=2 \mathrm{~ms}$ and $G_{x}=3.85 \mathrm{mT} \cdot \mathrm{m}^{-1}$, the expected cumulative broadening was close to $10 \mathrm{~Hz}$, which was much smaller than the $499 \mathrm{~Hz}$ bandwidth per pixel and which led to an effective spatial resolution of $4.1 \mathrm{~mm}$. Such broadening was safely neglected. Besides, following the generalization of the Bloch equations in nuclear magnetic resonance by $\mathrm{H}$. C. Torrey (38), one can show that, free diffusion being a random walk process, only magnitude is altered, and phase information is conserved. Provided the signal magnitude does not fall within the noise and diffusion is not restricted, the signal phase still carries relevant information for MRI - encoded by either the imaging gradients or the field inhomogeneities. Only the uncertainty on the phase increases when the SNR is reduced. For phase contrast MRI, even when motional narrowing occurs, data are impaired only during the effective encoding and reading times, whereas for amplitude-based methods the measured attenuation results from the integrated magnetic field history of each spin over the echo time, and diffusion smearing affect both the spatial resolution of the image and the values of the targeted parameter - such as $T_{2}^{*}$ or the apparent diffusion coefficients.

\section{Iron quantification}

In a first approximation, deposited aerosols can be seen as a collection of individual spherical droplets, which induce a set of dipolar magnetic fields. The resulting magnetic field perturbation at point $r$ is then given by the sum of these dipolar fields:

$$
\delta B(\mathbf{r})=\sum_{n=1}^{N} \frac{\mu_{0}}{4 \pi\left|\mathbf{r}-\mathbf{r}_{n}\right|^{3}}\left(3\left(\mathbf{m}_{n} \cdot\left(\mathbf{r}-\mathbf{r}_{n}\right)\right) \frac{\left(\mathbf{r}-\mathbf{r}_{n}\right)}{\left|\mathbf{r}-\mathbf{r}_{n}\right|^{2}}-\mathbf{m}_{n}\right)
$$

where $\mathbf{m}_{n}$ is the magnetic dipole moment of the $n^{\text {th }}$ droplet, which is located at $\mathbf{r}_{n}, \mu_{0}$ is the vacuum permeability, and $N$ is the number of droplets. The dipole moment $\mathbf{m}_{n}$ is parallel to the static magnetic field $\mathbf{B}_{0}=B_{0} \hat{\mathbf{e}}_{z}$. This moment is proportional to the mass of iron, $w_{n}$, contained within superparamagnetic contrast agent in the droplet: $\mathbf{m}_{n}=w_{n} m_{0} \hat{\mathbf{e}}_{z}$, where $m_{0}$ is the dipole moment of the ferrofluid per unit mass. Measured magnetic field perturbation maps correspond to the projection of $\delta \mathbf{B}(\mathbf{r})$ onto the $z$ axis:

$$
\delta B_{z}(\mathbf{r})=\sum_{n=1}^{N} w_{n} \frac{\mu_{0} m_{0}}{4 \pi\left|\mathbf{r}-\mathbf{r}_{n}\right|^{3}}\left(3 \frac{\left(\hat{\mathbf{e}}_{z} \cdot\left(\mathbf{r}-\mathbf{r}_{n}\right)\right)^{2}}{\left|\mathbf{r}-\mathbf{r}_{n}\right|^{2}}-1\right)
$$

To reconstruct the spatial distribution of iron-weighted droplets, i.e. their positions $\mathbf{r}_{n}$ and associated iron weights $w_{n}$, Equation [3] has to be inverted. The inversion problem may be made tractable by assuming that each voxel on the inner wall of the cast contains a single droplet of definite iron weight. At the MR spatial resolution this assumption is justified, as spatial variations of the magnetic field cannot be measured within a single voxel. Therefore, droplets are considered centered in voxels distributed over the inner wall of the cast. In this case, $N$ is the number of these inner wall voxels. The inversion problem is thus reduced to finding the iron weights $w_{n}(n \in[1, N])$. Equation [3] can be written as a matrix product:

$$
\Delta B_{z}=H \cdot W
$$

where $\Delta B_{z}$ is the $M$-dimensional vector representing the measured axial component of the magnetic field perturbation throughout the bulk voxels at positions $\mathbf{R}_{m}: \Delta B_{z_{m}}=\delta B_{z}\left(\mathbf{R}_{m}\right)$, $m \in[1, M], M$ being the number of internal bulk voxels; $W$ is the $N$-dimensional vector representing the iron weights $w_{n}, W_{n}=w_{n}$, $n \in[1, N]$; and $H$ is the $M \times N$-dimensional matrix with elements

$$
H_{m, n}=\frac{\mu_{0} m_{0}}{4 \pi\left|\mathbf{R}_{m}-\mathbf{r}_{n}\right|^{3}}\left(3 \frac{\left(\hat{e}_{z} \cdot\left(\mathbf{R}_{m}-\mathbf{r}_{n}\right)\right)^{2}}{\left|\mathbf{R}_{m}-\mathbf{r}_{n}\right|^{2}}-1\right)
$$

with $m \in[1, M]$ and $n \in[1, N]$. The phantom geometry sets the voxel positions $\mathbf{R}_{m}$ and $\mathbf{r}_{n}$, and thus the matrix $H$, while the measured magnetic field perturbation maps set the vector $\Delta B_{z}$. Since 
the number $N$ of boundary voxels is different from the number $M$ of bulk voxels, the matrix $H$ is not square and a direct inversion of Equation [4] is not possible. However, $N$ is in general smaller than $M$, so the number of unknowns is inferior to the number of equations. Equation [4] can thus be solved as a constrained optimization problem where the elements of $W$ must be positive. For the mouth-throat phantom, we have $N=1364$ and $M=2037$. Boundary voxels were calculated from a $3 D$ numerical version of the mouth-throat phantom downscaled to $(4 \times 4 \times 4) \mathrm{mm}^{3}$ resolution and registered to the MR dataset. All voxels directly connected to the phantom (i.e. the MR dataset) were taken as part of the phantom boundaries. The magnetic dipole moment per unit mass, $m_{0}=79 \times 10^{-3} \mathrm{~A} \cdot \mathrm{m}^{2} \cdot \mathrm{g}^{-1}$ at $1.5 \mathrm{~T}$. The constrained optimization problem was solved in MATLAB ${ }^{\circledR}$ (MathWorks, Natick, MA, USA) using a code developed in house.

\section{RESULTS}

\section{Aerosol characterization}

Figure 3 shows the mean cumulative and non-cumulative, normalized, mass-weighted size distribution diagrams for the aerosolized solution. The measured aerosol was polydisperse with $M M A D=(5.04 \pm 0.28) \mu \mathrm{m}$ and geometric standard deviation $1.78 \pm 0.01$.

\section{Single-photon emission computed tomography}

Figure 4 shows the three-dimensional distribution of deposited iron inferred from the recorded activity of deposited technetium- $99 \mathrm{~m}$. The maps are superimposed onto the coregistered $C T$ scan of the mouth-throat phantom. The calibrated sensitivity is $(3.5 \pm 0.5) \mathrm{Bq}$ per $\mathrm{ng}$ of iron per voxel where the mea- surement uncertainty is dominated by the collimator and detector blurring. In these specific experimental conditions, the SPECT detection limit is $0.15 \mathrm{ng}$ of iron per voxel. Activity was recorded not only along the cast wall but also within the cast volume and outside the cast. Most of the deposition is observed at the epiglottis, little in the pharynx and in the trachea, and none at the entrance of the mouth. The total mass of deposited iron integrated over the cast field of view is $(2.692 \pm 0.018) \mathrm{mg}$ for SPECT. Figure 7 later shows a projected map of deposited iron processed from the SPECT data on the 3D rendered volume of the mouth-throat phantom.

\section{Magnetic resonance imaging}

Globally in the phantom, $T_{1}^{[\mathrm{Fe}]}=318 \mathrm{~s}$ and $T_{1}=923 \mathrm{~s}$ in the presence and in the absence of iron oxide labeled aerosols, respectively. The signal magnitude over the four echo times is presented for the central slice of the phantom in the absence of deposited aerosols in Fig. 5(a) with respective maximal SNRs $80,61,57$, and 49 . In the presence of deposited aerosol, maximal SNRs are reduced to $46,39,34$, and 28 . The resulting $T_{2}^{*}$ map, shown in Fig. $5(\mathrm{~b})$, leads to $\left\langle T_{2}^{*}[\mathrm{Fe}]\right\rangle=(52 \pm 33) \mathrm{ms}$ with deposited aerosols in the cast whereas $\left\langle T_{2}^{*}\right\rangle=(70 \pm 41) \mathrm{ms}$ without deposited aerosols in the cast. The distribution of $T_{2}^{*}$ drops resulting from the aerosol deposition underlines that only $25 \%$ of the overall voxels exhibit $T_{2}^{*}$ shortening above $30 \mathrm{~ms}$ (Fig. 5(c)).

The computed static magnetic field perturbation maps, expressed in parts per million (ppm), are shown for eight central slices in Fig. 6(a). Corresponding phase shift errors are $0.03 \mathrm{rad}$ and $0.02 \mathrm{rad}$. Hence the deduced magnetic field perturbation error is $0.013 \mathrm{ppm}$. This sets the MRI detection limit, in the specific experimental conditions, close to $50 \mathrm{ng}$ of iron per voxel with an

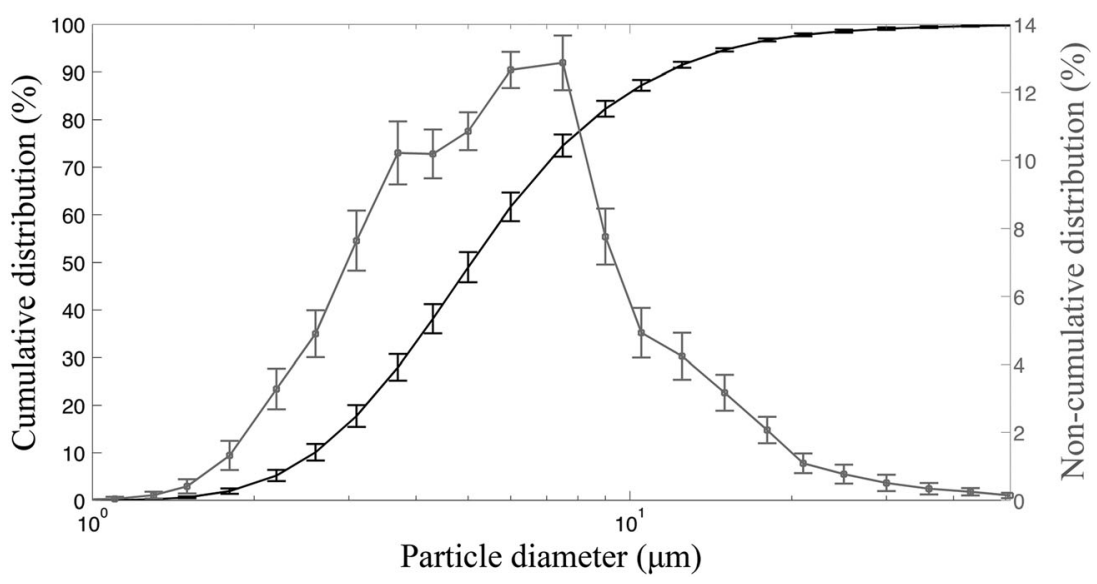

Figure 3. Measured cumulative (black) and non-cumulative (grey), normalized, mass-weighted size distribution diagrams for the nebulizer (DeVilbiss 35-B, Washington, DC, USA) with error bars representing one standard deviation around the mean $(n=3)$.

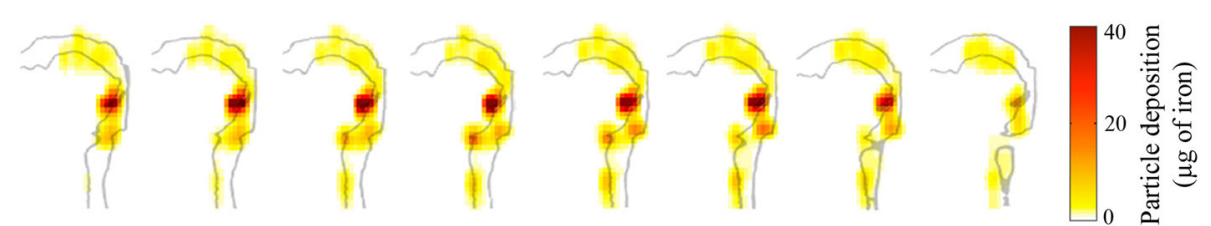

Figure 4. Three-dimensional maps of deposited aerosol (color-coded $\mu \mathrm{g}$ of iron) in the mouth-throat phantom computed from the SPECT activity maps. The maps are superimposed onto the co-registered CT scan of the mouth-throat phantom. Eight central slices are shown. 

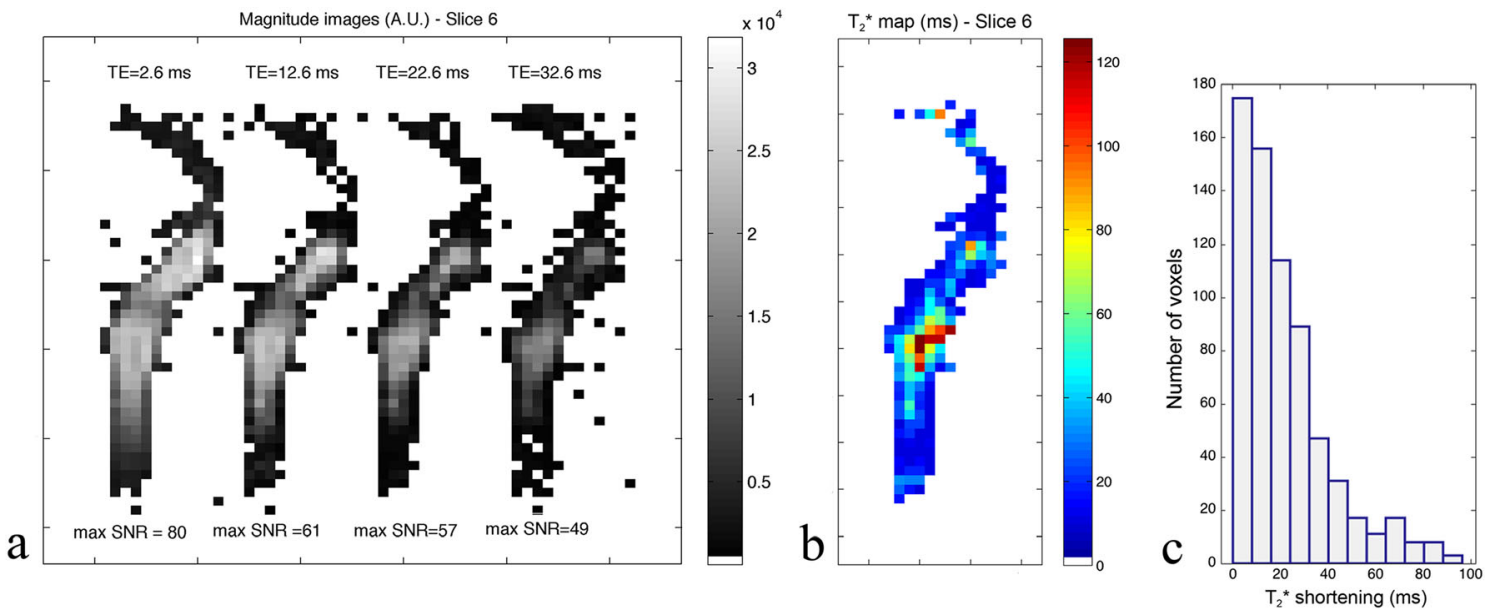

Figure 5. (a) Magnitude images of a central slice over the four acquired echo times, reported for the phantom with deposited aerosol (A.U.). (b) $T_{2}^{*}$ map in the corresponding slice computed from the four interleaved echoes (ms). (c) Volume distribution of $T_{2}^{*}$ shortening after aerosol deposition in the phantom (ms).

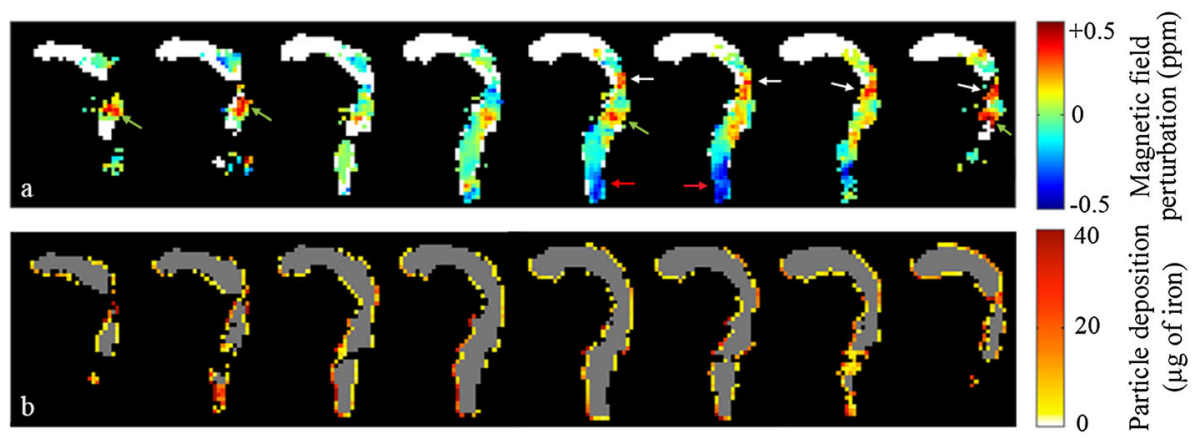

Figure 6. (a) Magnetic field variation maps (ppm) computed from the hyperpolarized helium-3 MRI phase shift maps in the mouth-throat phantom. Eight central slices are shown. The color-coded arrows show regions with high magnetic field variations resulting from iron-labeled aerosol deposition (white, epiglottis; green, larynx entrance; red, trachea). (b) Maps of deposited aerosol (color-coded $\mu \mathrm{g}$ of iron) in the mouth-throat phantom computed from the MRI static magnetic field perturbation maps. The maps are superimposed onto the numerical phantom (grey).

estimated sensitivity of 0.05 rad per ng of iron per voxel. Low signal magnitude was recorded at the upper and lower edges of the field of view, in the mouth area and at the trachea output, as the phantom reached regions of reduced coil sensitivity. Therefore, data at the mouth entrance were masked out due to low SNR therein. Elsewhere, static magnetic field perturbations point out the actual iron deposition. Main field perturbations reach $0.4 \mathrm{ppm}$ and $0.5 \mathrm{ppm}$ at the epiglottis and the trachea, respectively.

Figure 6(b) shows according to MRI the computed distribution of deposited iron on the interior wall of the phantom. Figure 7 shows the quantitative maps of deposited iron displayed along a surface projection method on the registered 3D model of the phantom. Main iron deposition sites are located at the epiglottis and down in the trachea. Significant deposition also occurs in the lower part of the pharynx. The total deposited iron mass is $(2.254 \pm 0.129) \mathrm{mg}$ for MRI.

\section{DISCUSSION}

The extrathoracic deposition of a nebulized, double-labeled solution was performed in a realistic mouth-throat phantom with both SPECT and hyperpolarized helium-3 MRI. Three-
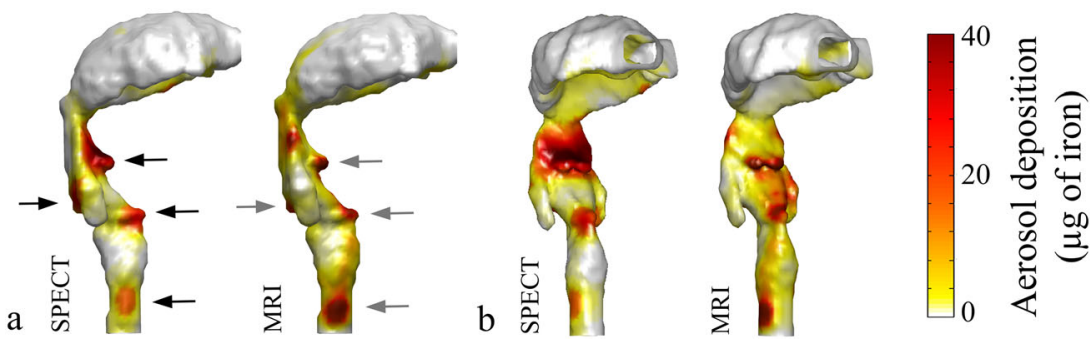

Figure 7. 3D rendered view, (a) side and (b) three-quarter, of deposited iron ( $\mu \mathrm{g}$ ) in the mouth-throat phantom computed from the SPECT activity maps and the MRI static magnetic field perturbation maps. The arrows show regions with higher particle deposition. 
dimensional distributions of the deposited aerosol were processed. The two modalities reveal large impaction zones at the epiglottis, at the entrance of the larynx and in the trachea. As expected (28), primary deposition occurs at the passage from the oropharynx to the larynx through the epiglottal constriction. Secondary deposition, spread over the larynx, ensues from the laryngeal jet flow, where particle velocity can reach $5 \mathrm{~m} \cdot \mathrm{s}^{-1}$ (39). Finally, turbulence downstream from the vocal folds leads to tertiary impaction in the trachea. Signal in the SPECT experiment was recorded outside of the phantom boundaries, underlining the broad point-spread function of the modality. The registration of the SPECT data onto the numerical phantom could not be perfect, as the cast mouth was slightly bent before imaging. However this affected only the mouth region, where little deposition occurred. The total iron mass inferred from helium-3 MRI is reasonably consistent with the SPECT outcome. The discrepancy may simply originate from an overestimation of the total amount of deposited iron due to the $11 \mathrm{~mm}$ FWHM point-spread function of the SPECT, which results in important blurring, and from lack of signal at the mouth from the effective MRI field of view (limited by the sensitivity of the coil used here). Overall, aerosol deposition has shifted by a few millimeters downwards at the epiglottis and in the trachea between SPECT and MRI acquisitions, possibly as a result of gravity over the two days allowed for activity decay.

SPECT is highly sensitive - its relative sensitivity is 2.6 times higher than it is for phase-contrast helium-3 MRI here - and it identifies main deposition areas in the anatomy, but it clearly cannot achieve the 3D spatial resolution of $(4 \times 4 \times 4) \mathrm{mm}^{3}$ expected from the $11 \mathrm{~mm}$ FWHM point-spread function of the system. Dedicated post-processing algorithms based on Monte Carlo simulations could be used to improve SPECT images (40-42) by partially removing radiation-induced blurring effects. Yet SPECT native spatial resolution remains intrinsically limited.

As shown here, particle deposition can be assessed with helium-3 MRI and iron oxide labeling through measurement of the inferred static magnetic field perturbations. The high sensitivity and the robustness of the technique rely on the phase measurement. It can be adjusted to the number of deposited iron particles by tuning the echo time in the acquisition sequence, though, for a given sensitivity, the dynamic range is limited by phase aliasing. Phase unwrapping algorithms could obviously be applied to recover a full range so long as spins in a voxel have not completely lost their phase coherence and the associated MR signal amplitude has not fallen into the noise. In the present work, the total acquisition time for reference and iron-weighted static magnetic field maps was $78 \mathrm{~s}$ each, but this could be easily reduced by at least an order of magnitude using an optimized single echo time sequence added with undersampling strategies and multiple-channel detection (43). MRI SNRs may be improved by more than one order of magnitude by administering a larger concentration of helium-3 with a polarization up to $70 \%$ such that the sensitivity of helium-3 phase-contrast MRI would exceed the SPECT level as well as that seen for standard MRI in (11). Ferromagnetic and superparamagnetic iron oxide particles are non-toxic and noncarcinogenic (44). They are deposited in the lungs through controlled voluntary inhalation for magnetopneumography at the level administered here (45). The acquisition time would scale to $80 \mathrm{~s}$ for a $(2 \times 2 \times 2) \mathrm{mm}^{3}$ spatial resolution as achieved for helium-3 MR flow measurement with high grade hyperpolarized helium-3 (38). For comparison, with the same radiation dose, the total acquisition time for SPECT - $32 \mathrm{~min}$ here for an effective spatial resolution of $11 \mathrm{~mm}$ - would be more than $1.5 \mathrm{~h}$ in an ultrahigh-resolution micro-SPECT (46), prohibiting in vivo imaging by long-run respiratory blurring and aerosol redistribution. Furthermore, the increased resolution would come at the expense of a reduced bore and thus a smaller field of view.

\section{CONCLUSION}

In the present work, hyperpolarized helium-3 MRI favorably compares with nuclear medicine in absolute quantification of aerosol deposition in a mouth-throat cast, providing high sensitivity and high spatial resolution within short acquisition times. In the future, aerosol deposition may be mapped in vivo in human airways during a single breath hold. It could also be mapped over several breathing cycles in order to follow the evolution of the deposition. Hence MR-based particle detection may be used as a new tool to study drug administration for inhalation therapies. More generally, phase-contrast helium-3 MRI may address fluid and particle dynamics in complex time-dependent geometries, otherwise experimentally inaccessible.

\section{Acknowledgements}

The authors would like to thank Aude Peudon, Department of Nuclear Medicine at Bicêtre Hospital, Bruno Louis, IMRB, Vicente Grau, Department of Engineering Science and Oxford eResearch, and Ludovic de Rochefort, IR4M, for their respective help in SPECT, fluid mechanics, registration and surface projection methods.

This work was initially funded by a national grant, ANR-07TecSan-005-01.

José Manuel Pérez Sánchez was funded within PHeLINet, a Marie Curie Actions Research Training Network, FP6 MRTN-CT2006-036002.

\section{REFERENCES}

1. Schanker LS. Drug absorption from the lung. Biochem. Pharmacol. 1978; 27: 381-385.

2. Patton JS, Fishburn CS, Weers JG. The lungs as a portal of entry for systemic drug delivery. Proc. Am. Thorac. Soc. 2004; 1: 338-344.

3. Mauroy B, Filoche M, Andrade J, Sapoval B. Interplay between geometry and flow distribution in an airway tree. Phys. Rev. Lett. 2003; 90: 148101.

4. Weibel ER, Sapoval B, Filoche M. Design of peripheral airways for efficient gas exchange. Respir. Physiol. Neurobiol. 2005; 148: 3-21.

5. Cryan S, Sivadas N, Garciacontreras L. In vivo animal models for drug delivery across the lung mucosal barrier. Adv. Drug Deliv. Rev. 2007; 59: 1133-1151.

6. Brand P, Rieger C, Schulz H, Beinert T, Heyder J. Aerosol bolus dispersion in healthy subjects. Eur. Respir. J. 1997; 10: 460-467.

7. DeHaan WH, Finlay WH. In vitro monodisperse aerosol deposition in a mouth and throat with six different inhalation devices. J. Aerosol Med. 2001; 14: 361-367.

8. Snell NJ, Ganderton D. Assessing lung deposition of inhaled medications. Consensus statement from a workshop of the British Association for Lung Research, held at the Institute of Biology, London, U. K. Respir. Med. 1999; 93: 123-133.

9. Farr SJ, Warren SJ, Lloyd P, Okikawa JK, Schuster JA, Rowe AM, Rubsamen RM, Taylor G. Comparison of in vitro and in vivo efficiencies of a novel unit-dose liquid aerosol generator and a pressurized metered dose inhaler. Int. J. Pharm. 2000; 198: 63-70.

10. Clark A, Kuo M-C, Newman S, Hirst P, Pitcairn G, Pickford M. A comparison of the pulmonary bioavailability of powder and liquid aerosol formulations of salmon calcitonin. Pharm. Res. 2008; 25: 1583-1590. 
11. Martin AR, Thompson RB, Finlay WH. MRI Measurement of regional lung deposition in mice exposed nose-only to nebulized superparamagnetic iron oxide nanoparticles. J. Aerosol Med. Pulm. Drug Deliv. 2008; 21: 335-342.

12. de Rochefort L, Nguyen T, Brown R, Spincemaille P, Choi G, Weinsaft J, Prince MR, Wang Y. In vivo quantification of contrast agent concentration using the induced magnetic field for time-resolved arterial input function measurement with MRI. Med. Phys. 2008; 35: 5328-5339.

13. Foster-Gareau P, Heyn C, Alejski A, Rutt BK. Imaging single mammalian cells with a 1.5 T clinical MRI scanner. Magn. Reson. Med. 2003; 49: 968-971.

14. Hinds KA, Hill JM, Shapiro EM, Laukkanen MO, Silva AC, Combs CA, Varney TR, Balaban RS, Koretsky AP, Dunbar CE. Highly efficient endosomal labeling of progenitor and stem cells with large magnetic particles allows magnetic resonance imaging of single cells. Blood 2003; 102: 867-872.

15. Branca RT, Cleveland ZI, Fubara B, Kumar CSSR, Maronpot RR, Leuschner C, Warren WS, Driehuys B. Molecular MRI for sensitive and specific detection of lung metastases. Proc. Natl. Acad. Sci. U. S. A. 2010; 107: 3693-3697.

16. de Rochefort L, Brown R, Prince MR, Wang Y. Quantitative MR susceptibility mapping using piece-wise constant regularized inversion of the magnetic field. Magn. Reson. Med. 2008; 60: 1003-1009.

17. de Rochefort L, Liu T, Kressler B, Liu J, Spincemaille P, Lebon V, Wu J, Wang Y. Quantitative susceptibility map reconstruction from MR phase data using bayesian regularization: validation and application to brain imaging. Magn. Reson. Med. 2009; 63: 194-206.

18. Mills PH, Wu Y-JL, Ho C, Ahrens ET. Sensitive and automated detection of iron-oxide-labeled cells using phase image cross-correlation analysis. Magn. Reson. Imaging 2008; 26: 618-628.

19. MacFall JR, Charles HC, Black RD, Middleton H, Swartz JC, Saam B, Driehuys B, Erickson C, Happer W, Cates GD, Johnson GA, Ravin CE. Human lung air spaces: potential for $M R$ imaging with hyperpolarized He-3. Radiology 1996; 200: 553-558.

20. Bachert $P$, Schad LR, Bock M, Knopp MV, Ebert M, Grossmann T, Heil W, Hofmann D, Surkau R, Otten EW. Nuclear magnetic resonance imaging of airways in humans with use of hyperpolarized ${ }^{3} \mathrm{He}$. Magn. Reson. Med. 1996; 36: 192-196.

21. Kauczor HU, Surkau R, Roberts T. MRI Using hyperpolarized noble gases. Eur. Radiol. 1998; 8: 820-827.

22. Salerno M, Altes TA, Brookeman JR, de Lange EE, Mugler JP III. Dynamic spiral MRI of pulmonary gas flow using ${ }^{3} \mathrm{He}$ : preliminary studies in healthy and diseased lungs. Magn. Reson. Med. 2001; 46: 667-677.

23. Moller HE, Chem XJ, Saam B, Hagspiel KD, Johnson GA, Altes TA, de Lange EE, Kauczor HU. MRI of the lungs using hyperpolarized noble gases. Magn. Reson. Med. 2002; 47: 1029-1051.

24. de Lange EE, Mugler JP, Brookeman JR, Knight-Scott J, Truwit JD, Teates CD, Daniel TM, Bogorad PL, Cates GD. Lung air spaces: MR imaging evaluation with hyperpolarized ${ }^{3} \mathrm{He}$ gas. Radiology 1999; 210 : 851-857.

25. Guenther D, Eberle B, Hast J, Lill J, Markstaller K, Puderbach M, Schreiber WG, Hanisch G, Heussel CP, Surkau R, Grossmann T, Weiler N, Thelen M, Kauczor $\mathrm{H}-\mathrm{U} .{ }^{3} \mathrm{He} \mathrm{MRI}$ in healthy volunteers: preliminary correlation with smoking history and lung volumes. NMR Biomed. 2000; 13: 182-189.

26. Viallon $M$, Berthezène $Y$, Décorps $M$, Wiart $M$, Callot $V$, Bourgeois $M$, Humblot $H$, Briguet $A$, Crémilleux $Y$. Laser-polarized ${ }^{3} \mathrm{He}$ as a probe for dynamic regional measurement of lung perfusion and ventilation using magnetic resonance imaging. Magn. Reson. Med. 2000; 44: 1-4.
27. Dimitrov IE, Insko E, Rizi R, Leigh JS. Indirect detection of lung perfusion using susceptibility-based hyperpolarized gas imaging. J. Magn. Reson. Imaging 2005; 21: 149-155.

28. de Rochefort L, Maître X, Fodil R, Vial L, Louis B, Isabey D, Croce C, Darrasse L, Apiou G, Caillibotte G, Bittoun J, Durand E. Phase-contrast velocimetry with hyperpolarized $3 \mathrm{He}$ for in vitro and in vivo characterization of airflow. Magn. Reson. Med. 2006; 55: 1318-1325.

29. Fain S, Korosec FR, Holmes JH, O'Halloran R, Sorkness RL, Grist TM. Functional lung imaging using hyperpolarized gas MRI. J. Magn. Reson. Imaging 2007; 25: 910-923.

30. Tsai LL, Mair RW, Rosen MS, Patz S, Walsworth RL. An open-access, very-low-field MRI system for posture-dependent ${ }^{3} \mathrm{He}$ human lung imaging. J. Magn. Reson. 2008; 193: 274-285.

31. van Beek EJ, Wild JM, Kauczor HU, Schreiber W, Mugler JP 3rd, de Lange EE. Functional MRI of the lung using hyperpolarized 3helium gas. J. Magn. Reson. Imaging 2004; 20: 540-554.

32. Grgic B, Finlay WH, Burnell PKP, Heenan AF. In vitro intersubject and intrasubject deposition measurements in realistic mouth-throat geometries. J. Aerosol Sci. 2004; 35: 1025-1040.

33. Grgic B, Finlay WH, Heenan AF. Regional aerosol deposition and flow measurements in an idealized mouth and throat. J. Aerosol Sci. 2004; 35: $21-32$.

34. Sandeau J, Katz I, Fodil R, Louis B, Apiou-Sbirlea G, Caillibotte G, Isabey D. CFD simulation of particle deposition in a reconstructed human oral extrathoracic airway for air and helium-oxygen mixtures. J. Aerosol Sci. 2010; 41: 281-294.

35. Choukeife J, Maître X, Tastevin G, Nacher PJ. On-site production of hyperpolarized helium-3 gas for lung MRI. Proc. Int. Soc. Magn. Reson. Med. 2003; 1391: 280.

36. Liner J, Weissman S, Determination of the temperature dependence of gaseous diffusion coefficients using gas chromatographic apparatus. J. Chem. Phys. 1972; 56(5): 2288-2290.

37. Callaghan PT, Eccles CD. Sensitivity and resolution in NMR imaging. J. Magn. Reson. 1987; 71(3): 426-445.

38. Torrey HC. Bloch equations with diffusion terms. Phys. Rev. 1956; 104(3): 563-565.

39. Maître X, Sandeau J, Fodil R, Louis B, Sarracanie M, Santarelli R, Durand E, Darrasse L, Caillibotte G, Bittoun J, Isabey D. Hyperpolarized gas velocity mapping to capture jet flows generated in a human upper airway constriction: a phantom study. Proc. Eur. Soc. Magn. Reson Med. Biol. 2008; 262: 170.

40. Andreo P. Monte Carlo techniques in medical radiation physics. Phys. Med. Biol. 2000; 36: 861-920.

41. Raeside DE. Monte Carlo principles and applications. Phys. Med. Biol. 2002; 21: 181-197.

42. Ljungberg M, Strand S-E, King MA. Monte Carlo Calculations in Nuclear Medicine: Applications in Diagnostic Imaging, 2nd edn. CRC Press: Boca Raton, FL, 2012.

43. Meise FM, Rivoire J, Terekhov M, Wiggins GC, Keil B, Karpuk S, Salhi Z, Wald LL, Schreiber LM. Design and evaluation of a 32-channel phased-array coil for lung imaging with hyperpolarized 3-helium. Magn. Reson. Med. 2010; 63: 456-464.

44. Stokinger HE. A review of world literature finds iron oxides noncarcinogenic. Am. Ind. Hyg. Assoc. J. 1984; 45: 127-133.

45. Cohen D, Arai SF, Brain JD. Smoking impairs long-term dust clearance from the lung. Science 1979; 204: 514-517.

46. Meng LJ, Fu G, Roy EJ, Suppe B, Chen CT. An ultrahigh resolution SPECT system for I-125 mouse brain imaging studies. Nucl. Instrum. Methods Phys. Res. A 2009; 600: 498-505. 